\title{
Periodogram Connectivity of EEG Signals for the Detection of Dyslexia
}

\author{
F. J. Martinez-Murcia, A. Ortiz, R. Morales-Ortega, P. J. López, J. L. Luque, D. Castillo-Barnes, F. \\ Segovia, I. A. Illan, J. Ortega, J. Ramirez, J. M. Gorriz
}

\begin{abstract}
Electroencephalography (EEG) signals provide an important source of information of brain activity at different areas. This information can be used to diagnose brain disorders according to different activation patterns found in controls and patients. This acquisition technology can be also used to explore the neural basis of less evident learning disabilities such as Developmental Dyslexia (DD). DD is a specific difficulty in the acquisition of reading skills not related to mental age or inadequate schooling, whose prevalent is estimated between $5 \%$ and $12 \%$ of the population. In this paper we propose a method to extract discriminative features from EEG signals based on the relationship among the spectral density at each channel. This relationship is computed by means of different correlation measures, inferring connectivity-like markers that are eventually selected and classified by a linear support vector machine. The experiments performed shown AUC values up to 0.7 , demonstrating the applicability of the proposed approach for objective DD diagnosis.
\end{abstract}

\section{Keywords}

Periodogram, EEG, Connectivity, Principal Component Analysis, Dyslexia 